Check for updates

Cite this: RSC Adv., 2018, 8, 17373

Received 18th November 2017 Accepted 3rd May 2018

DOI: $10.1039 / c 7 r a 12550$

rsc.li/rsc-advances

\title{
Metal-free heterogeneous and mesoporous biogenic graphene-oxide nanoparticle-catalyzed synthesis of bioactive benzylpyrazolyl coumarin derivatives $\dagger$
}

\author{
T. A. J. Siddiqui, ${ }^{a}$ Balaji G. Ghule, (D) ${ }^{\text {b }}$ Shoyebmohamad Shaikh, ${ }^{b}$ \\ Pritamkumar V. Shinde, (D) ${ }^{b}$ Krishna Chaitanya Gunturu, ${ }^{a}$ P. K. Zubaidha, \\ Je Moon Yun, (D) Colm O'Dwyer, (D)*de Rajaram S. Mane (D)*bc \\ and Kwang Ho Kim (D) *c
}

\begin{abstract}
We report the preparation of graphene oxide nanoparticles (GONPs), a metal-free, heterogeneous, nontoxic, reusable and mesoporous green-(acid)-catalyst obtained by sugar carbonization through a microwave chemical synthesis method for the synthesis of bio-active benzylpyrazolyl coumarin derivatives (BCDs) under thermal conditions $\left(50^{\circ} \mathrm{C}\right)$ in ethanol solvent. The obtained products were purified by recrystallization from ethanol, assuring usability of GONPs in multicomponent reactions (MCRs) that could find wide application in the synthesis of a variety of biologically potent molecules of therapeutic significance.
\end{abstract}

\section{Introduction}

New facets of conventional heterogeneous catalysts have stimulated extensive efforts in nanochemistry methods for preparing new catalytic materials with greater precision. ${ }^{1}$ The most widely explored non-noble and platinum group catalytic nanomaterials include transition metals, metal oxides, ${ }^{2}$ and hybrid materials based on inorganic and enzymes ${ }^{3-7}$ where the use of ultraviolet-visible irradiation is often obligatory. The development of metal-free green synthesis of fine chemicals and pharmaceuticals ${ }^{8-10}$ to overcome the shortcomings of transition metal-based catalysts (both heterogeneous and homogenous $)^{\mathbf{1 1 , 1 2}}$ used in the catalytic process finds at niche place in the industrial community. ${ }^{13,14}$ As such, the search for catalysts that combine the toxicological benefits of a metal-free synthesis with the amenities of heterogeneous work-up, whilst

${ }^{a}$ School of Chemical Sciences, SRTM University, Nanded, India-431606

${ }^{b}$ Center for Nanomaterials \& Energy Devices, School of Physical Sciences, SRTM University, Nanded, India-431606. E-mail: rajarammane70@srtmun.ac.in

${ }^{c}$ Global Frontier R\&D Center for Hybrid Interface Materials, Pusan National University, San 30 Jangjeon-dong, Geumjeong-gu, Busan, 609-735, Republic of Korea.E-mail: kwhokim@pusan.ac.kr

${ }^{d}$ School of Chemistry, University College Cork, Cork T12 YN60, Ireland. E-mail: c. odwyer@ucc.ie

${ }^{e}$ Micro-nano Systems Centre, Tyndall National Institute, Lee Maltings, Cork T12 R5CP, Ireland

$\dagger$ Electronic supplementary information (ESI) available: Quantitative analysis data of energy-dispersive X-ray spectroscopy spectrums, deconvolution of GONPs XPS C1s peak, structural analysis data of ${ }^{1} \mathrm{H}$ NMR, ${ }^{13} \mathrm{C}$ NMR and mass spectra and their respective spectra. See DOI: $10.1039 / \mathrm{c} 7 \mathrm{ra12550j}$ having high activity, is a continuing effort of censorious importance. ${ }^{15,16}$ Based on abundant elements, finding alternative metal-free hetero or homogeneous, selective or high performance catalysts is alluring and important. The potential of carbon materials (CMs) as heterogeneous catalysts could be profound. The term "carbocatalysis" is used to denote the utilization of metal-free carbon materials as direct/active catalysts rather than inert catalysts. Certain carbonaceous materials have intrinsic physicochemical properties such as exclusive structure, gigantic surface area, good pore interconnectivity and high chemical/thermal stability. The CMs such as activated carbon, fullerene, single and multi-walled carbon nanotubes, and graphene oxide (GO) etc., have been manifested as efficient carbocatalyst. CMs offer new opportunities for the development of advanced carbon-based nanocatalysts with much improved catalytic performance, due to their peculiar molecular structures and optoelectronic properties. Among them, GO is highly preferred due to its low-cost, 2D structure, rich chemical functionality (solid-acid, solid-base, redox and defect sites) and chemical stability and mechanical robustness. To date, several metal-based catalytic organic transformations have been replaced by GO-based carbocatalytic systems. ${ }^{17-21}$ To the best of our knowledge, the majority of GO sheets reported were obtained by the excruciating methods, ${ }^{22-24}$ typically carried out by the use of fuming nitric acid, concentrated sulfuric acid, potassium chlorate, potassium permanganate and hydrogen peroxide etc. Furthermore, most of the experimental processes generate waste gas, large amounts of acidic water and a little nitrogen and sulphur as well as metal ion co-doped GO sheets, 

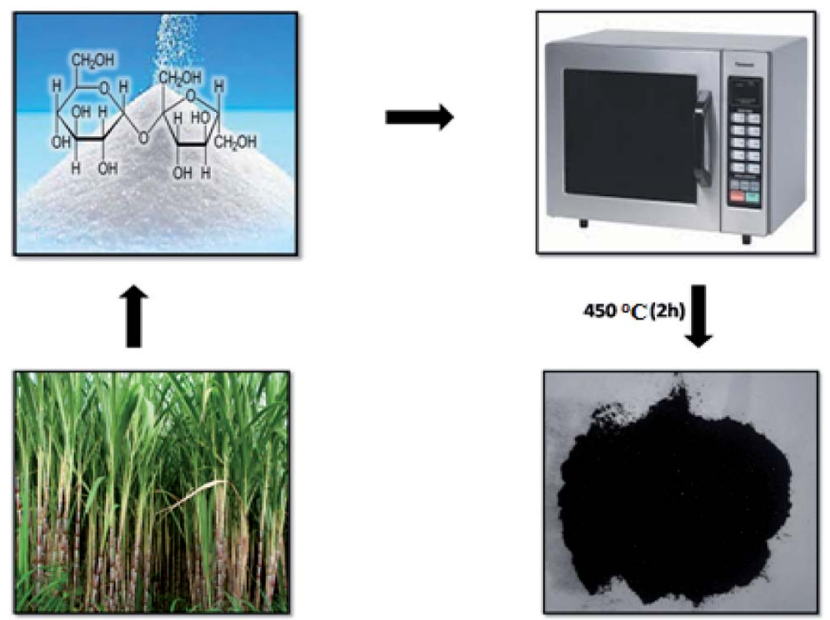

Scheme 1 A micro-oven-assisted chemical method used for obtaining GONPs from market quality sugar.

implying the presence of environmental and health-related issues with processing and use. Therefore, current scenarios require the organic chemist to systematize the research in such a way that it preserves the environment and to develop procedures that are both eco-friendly and economically suitable. One major objective is, therefore, to simplify and accommodate the traditional procedures in a modern way with the aim of minimizing pollution effects to the least. ${ }^{25,26}$ In continuation of research on the synthesis of highly functionalized BCDs, ${ }^{27-30}$ here, we report an unveiling protocol for the preparation of the GONPs which has specific area $\sim 5.15 \mathrm{~m}^{2} \mathrm{~g}^{-1}$, mean pore diameter $\sim 4.6 \mathrm{~nm}$ with pore volume of $\sim 0.0876 \mathrm{~cm}^{3} \mathrm{~g}^{-1}$ (ref. 31) and their catalytic applications as novel and stable heterogeneous catalyst for the synthesis of BCDs. Environmental prestige, economic soul and recyclability are the advantages of this novel catalyst. In a nutshell, synthesis of microwave-mediated GONPs on market quality sugar carbonation was carried out by irradiating radiations at $400-500 \mathrm{~W}$ power for $10-15 \mathrm{~min}$ duration. The synthesized product was air-annealed at $450{ }^{\circ} \mathrm{C}$ for $2 \mathrm{~h}$ and the black-product was obtained i.e. GONPs (Scheme 1). The GONPs were characterized for their structure, morphology, grain-size, phase-purity, porosity, pore-size distribution, elemental composition and catalytic activity.

\section{Experimental details}

\section{Chemicals}

All the reagents were purchased from Merck and Spectrochem and were used without further purification. The reaction was monitored by TLC using $0.2 \mathrm{~mm}$ Merck silica gel 60 F254 precoated plates, which were seen with UV light. Melting points were measured by open capillary method.

\section{Synthesis of GONPs through market quality sugar}

Dry table-sugar was crushed in a mortar to form uniform powder and then transferred into a glass beaker and heated under 400-500 $\mathrm{W}$ power radiations for 4-6 times with each of 12 min duration in a microwave oven. The formed charcoal was collected and crushed in a mortar pastor to get smooth powder and annealed at $450^{\circ} \mathrm{C}$ for $2 \mathrm{~h}$. The sample formed is black in appearance and smooth in nature. This black powder was insoluble in water, ethanol, methanol, and benzene but, partially soluble in dimethyl sulphoxide. The annealed powder was collected and well characterized by the X-ray diffraction (XRD, D8-Discovery Bruker, $\mathrm{Cu} \mathrm{K} \alpha, 40 \mathrm{kV}, 40 \mathrm{~mA}$ ) pattern, scanned from 20 to $80^{\circ}$. The Raman spectrum was recorded on a WITec system within a Raman shift of $1000-2000 \mathrm{~cm}^{-1}$. Fieldemission scanning electron microscopy (FE-SEM, Hitachi, S$4800,15 \mathrm{kV}$ ), energy dispersive X-ray (EDX) analysis, and highresolution transmission electron microscopy (HR-TEM) along with the selected area electron diffraction (SAED) pattern (Technai F20) were used to observe the surface morphology, grain size, and elemental configuration, respectively. X-ray photoelectron spectroscopy (XPS, VG Scientific ESCALAB250) was utilized to analyze the chemical composition and bonding status. The Brunauer-Emmett-Teller surface area was obtained using a Micrometrics ASAP2010 analyzer in a $\mathrm{N}_{2}$ environment with proper moisture removal from the sample.

\section{General procedure for the preparation of benzylpyrazoly coumarin derivatives}

To a mixture of $20 \%$ of catalyst and $3 \mathrm{ml}$ of ethanol as a solvent was added phenyl hydrazine $1(1 \mathrm{mmol})$, ethyl acetoacetate $2(1$ $\mathrm{mmol})$, aromatic aldehyde $(1 \mathrm{mmol})$ and 4-hydroxycoumarin 4 $(1 \mathrm{mmol})$ and heated at $50{ }^{\circ} \mathrm{C}$. The clear reaction mixture was stirred for the stipulated time and the mixture becomes turbid as the reaction proceeds. After completion of the reaction (monitored by TLC), the free-flowing solid was dissolved in dichloromethane to separate the heterogeneous catalyst by simple filtration method. Afterwards, the filtrate was evaporated under reduced pressure to obtain crude products as pale-yellow solids. The products, thus, obtained was recrystallized from hot ethanol to the get pure compounds as white or pale-yellow solids. Yields were calculated after recrystallization (Scheme 2). The isolated compounds were well characterized by ${ }^{1} \mathrm{H} \mathrm{NMR}$, ${ }^{13} \mathrm{C}$ NMR, and Mass spectrum and reported in supportive information.

\section{Results and discussion}

Fig. 1a shows the X-ray diffraction pattern of as-prepared GONPs, which is in accordance with (002) and (100) planes of graphitic carbon (JCPDF\# 41-1487). The Raman shift spectrum (Fig. 1b) reveals the presence of two characteristic bands of carbon at $1352 \mathrm{~cm}^{-1}$ (D-band) for the $\mathrm{sp}^{3}$ configuration and

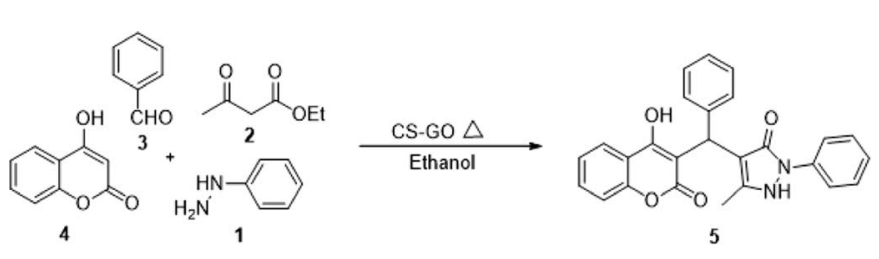

Scheme 2 Green synthesis of BCDs. 
$1592 \mathrm{~cm}^{-1}$ (G-band) for the $\mathrm{sp}^{2}$ graphitic configuration. The 0.75 intensity ratio of the D-band over the G-band signifies the graphitic behavior of GONPs. ${ }^{32}$ The Fourier-transform infrared (FTIR) spectrum of GONPs (Fig. 1c) confirms basal planes of $\mathrm{sp}^{2}$ carbon functionalized with hydroxyl $(-\mathrm{OH})$, epoxide $(\mathrm{C}-\mathrm{O}-\mathrm{C})$ and carboxylic acid $(-\mathrm{COOH})$ groups ${ }^{33,34}$ qualitatively demonstrating the presence of GO rather other carbonaceous phases such as graphite and graphene. The FTIR spectrum shows broad and strong bands at $\sim 3390$ and $1090 \mathrm{~cm}^{-1}$ which are attributed to stretching vibrations and in plane bending vibration of $-\mathrm{OH} / \mathrm{H}_{2} \mathrm{O}$. A weak band at $\sim 2926 \mathrm{~cm}^{-1}$ corresponds to the stretching vibrations of the $\mathrm{C}-\mathrm{H}$ bond. Presence of the featured bands at $\sim 1622 \mathrm{~cm}^{-1}$ and $1716 \mathrm{~cm}^{-1}$ confirms the stretching of $\mathrm{C}=\mathrm{O}$. A relatively sharp band at $\sim 1205 \mathrm{~cm}^{-1}$ is due to stretching of $-\mathrm{C}-\mathrm{O}$ or bending of alkyl groups. Band appeared at $618 \mathrm{~cm}^{-1}$ was assigned to $\mathrm{CH}$ deformation. The field-emission scanning electron microscopy image of GONPs exhibits crystallites of irregular dimensions (Fig. 1d). The globular GONPs are typically interconnected to one another at the edges. The $72: 28$ composition ratio for carbon and oxygen elements supports a GO composition (Fig. S1 $\dagger$ ). Fig. 1e and $f$ presents digital images of normal and high-resolution transmission electron microscopy where, surprisingly, irregular grains of 10-50 $\mathrm{nm}$ diameters are found. Selected area electron diffraction pattern in Fig. 1f demonstrates the nanocrystalline nature of GONPs. ${ }^{35,36}$ Consistent with X-ray diffraction results, the interplanar distances of $\sim 0.38 \mathrm{~nm}$ (inset of Fig. 1f) and $\sim 0.28 \mathrm{~nm}$ for lattice fringe widths of the (002) and (100) planes, respectively, are corroborated.

A steep-rise in the nitrogen/adsorption curve is observed after a relative pressure $\left(p / p_{\mathrm{o}}\right)>0.3$, signifying (Fig. 1g) the mesoporous nature of GONPs. Correspondingly derived surface-area and micro-pore surface-area of GONPs are 5.15 and $33.12 \mathrm{~m}^{2} \mathrm{~g}^{-1}$, respectively. The pore-size distribution curve (Fig. 1h) of GONPs reveals a total specific pore-volume and average pore-diameter of $0.0876 \mathrm{~cm}^{3} \mathrm{~g}^{-1}$ and $46.13 \AA$, respectively. The deconvoluted high resolution C1s photoemission peak from GONPs by XPS is shown in Fig. S2. $\dagger$ The calibration of carbon C1s core-level of adventitious was $284.6 \mathrm{eV}$, corresponding to the $\mathrm{sp}^{2}$ carbon phase. The $\mathrm{C} 1 \mathrm{~s}$ peaks were fitted with three contributions: $\mathrm{sp}^{2}(284.6 \mathrm{eV}), \mathrm{C}-\mathrm{O}(286.8 \mathrm{eV}), \mathrm{C}=\mathrm{O}$ (288.9 eV), respectively.

The amount of oxygen in the GONPs can be explained by bonding of oxygen to the defected carbon bonds. The abovementioned measurements have provided evidence that our microwave-assisted product is structurally close to GO. Taking into consideration the importance of carbon-based materials, ${ }^{35,36}$ the catalytic behaviour of the prepared GONPs was studied for the synthesis of BCDs.

Design and development of interweaved molecular scaffolds for the biological applications have fascinated much attention as these moieties exhibit wide-ranging and improved bioactivities. It is anticipated that the integration of the 3-benzyl coumarin and pyrazolone moieties leading to benzylpyrazolyl coumarin scaffolds could be fascinating and beneficial from a biological point of view. ${ }^{27-30}$ Amongst few earlier reports for this scaffold, the use of glacial acetic acid in water under reflux
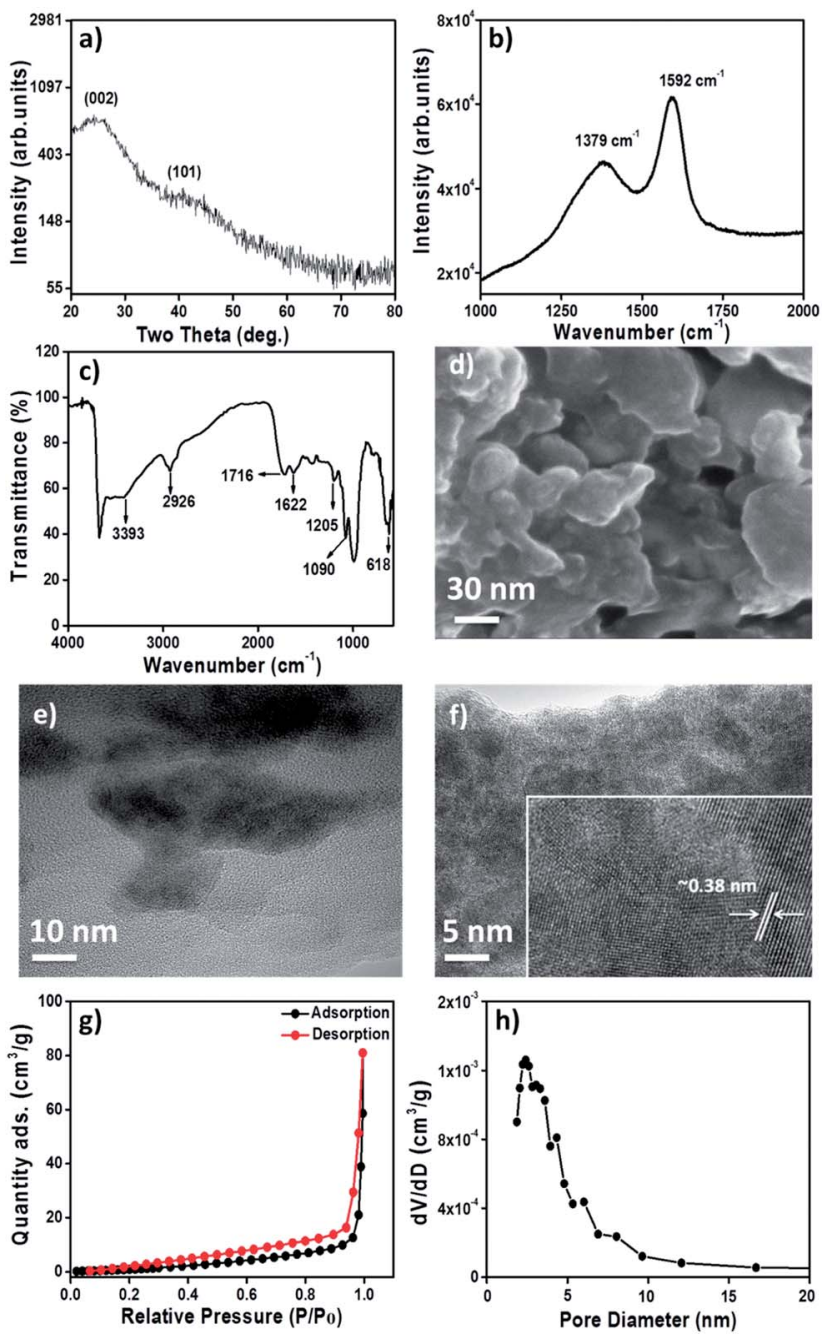

Fig. 1 (a) X-ray diffraction pattern, (b) Raman spectrum (concluding graphitic nature), (c) FTIR spectrum, (d) FE-SEM, (e) TEM (showing interconnected and irregular signature), (f) HR-TEM (inset shows $0.38 \mathrm{~nm}$ interplanar spacing of (002) plane), (g) adsorption-desorption isotherm, and (h) pore-size distribution curve (confirming the mesoporous character) of GONPs.

conditions along with molecular motif of scaffold explored by single X-ray crystallographic analysis ${ }^{27}$ and metal containing catalysts were explored..$^{28-30}$ Natural carbon was demonstrated as an alternative to metal and metal oxide-based catalysts due to its worldwide natural abundance, stability, inexpensiveness and non-toxic signature. ${ }^{\mathbf{1 9 , 2 0}}$ As part of our interest in exploring environmentally benign GONPs and other materials as greencatalysts for the synthesis of several biologically important heterocyclic compounds, we report a facile one-pot MCR for the synthesis of various BCDs in ethanol solvent using GONPs derived from natural sugar. The effects of solvent, catalyst amount, reaction duration and molar-ratio of the raw material on the yield of BCDs were studied systematically. The synthesis of BCDs 4-[(4-hydroxy-2-oxo-2H-chromen-3-yl)-phenylmethyl]-5methyl-2-phenyl-1,2-dihydro-pyrazol-3-one with high percent yield by one-pot four-component condensation reactions is one of the major objectives of this work. These four-components i.e. 
Table 1 A Four-component reactions was done with different derivatives of aldehydes in the presence of catalytic amount of GONPs

Sr. no Aromatic aldehydes

Time (h)

Product (BCDs)

Yield (\%)

1

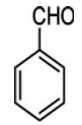

6

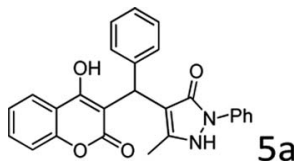

90

$3 a$

CHO

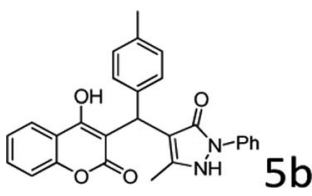

$3 b$

5

93<smiles>O=Cc1ccc(F)cc1</smiles>

8

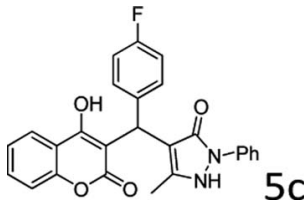

87

$3 c$

3

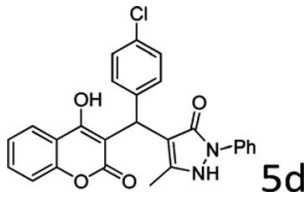

90

3d

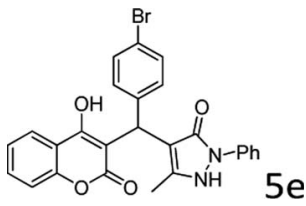

93

10
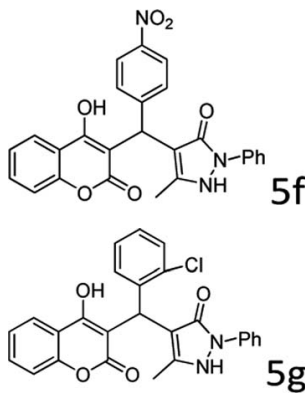

86<smiles>Cc1[nH]n(-c2ccccc2)c(=O)c1C(c1ccccc1[N+](=O)[O-])c1c(O)c2ccccc2oc1=O</smiles>

$3 \mathrm{~h}$

10<smiles>O=Cc1cccc(Br)c1</smiles>

7

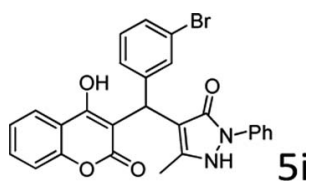

10

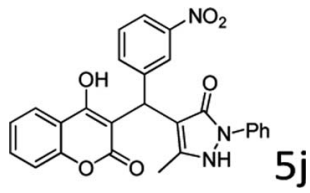

88<smiles>O=Cc1cccc([N+](=O)[O-])c1</smiles>

3j
Furthermore, the optimization study of this four-component reaction was studied in different solvents (Table 2). The best results were obtained in ethanol solvent, while water as solvent 
results in a sticky product with unreacted starting material. The reaction without GONPs catalyst could not provide the product even after $10 \mathrm{~h}$ under identical conditions (see entry 1 of Table 2). The optimized yield of the product (5) was obtained in ethanol as a solvent medium using $20 \%$ of GONPs as catalyst (see 10 entry of Table 2). As envisaged, the GONPs displayed excellent catalytic properties due to their considerable surface area, abundant active sites and unique chemical/physical properties. The functional groups (hydroxy, epoxide and carboxyl etc.) as seen in FTIR studies endow suitable acidity and oxidizing properties providing high tenability of ethanol solvent towards polar organic substrates and reagents. The ethanol solvent polarity created ambient conditions for good conversion, which was further optimized for the reaction purity, yield and speed. Under optimized conditions in ethanol solvent, as high as $93 \%$ yield of BCD was obtained. Having set the reaction conditions, the protocol was studied with a variety of substituted benzaldehydes. Equimolar amounts of substituted of phenyl hydrazine (1), ethyl acetoacetate (2), aromatic aldehyde (3), 4-hydroxycoumarin (4), and $20 \%$ of catalyst under thermal condition $\left(50{ }^{\circ} \mathrm{C}\right)$ in solvent ethanol (Scheme 2) have provided a library of BCD (5) in good to excellent yields (8693\%) (Table 1). The reaction proceeded smoothly and tolerated electron withdrawing and electron donating substituted benzaldehydes.

The reactions were consistently carried out at $1 \mathrm{mmol}$ scale and no change of product yield was observed when scaled up to the $10 \mathrm{mmol}$ scale. It was encouraging to find that the final products (5) could be isolated by simple filtration, avoiding any requirement for chromatographic separation. This optimized reaction condition was applied to different aldehydes to check the general applicability and functional group tolerance. The reaction proceeds well to give excellent yields of the product without any column purification. The catalyst was easily removed from the reaction mixture due to its heterogeneous character. Furthermore, their purity was so high, no preparation in an analytically pure form by single recrystallization using ethanol as a solvent was necessary. The final products were confirmed by elemental analysis, IR, ${ }^{1} \mathrm{H}$ NMR, ${ }^{13} \mathrm{C}$ NMR and HRMS analytical and spectral techniques (SI-1).

Table 2 Optimization of catalyst with different solvents for the synthesis of CBDs (5)

\begin{tabular}{llllll}
\hline Sr. no. & $\begin{array}{l}\text { Catalyst } \\
(\%)\end{array}$ & Solvent & $\begin{array}{l}\text { Temperature } \\
\left({ }^{\circ} \mathrm{C}\right)\end{array}$ & $\begin{array}{l}\text { Time } \\
(\mathrm{h})\end{array}$ & $\begin{array}{l}\text { Yield } \\
(\%)\end{array}$ \\
\hline 1 & $\begin{array}{l}\text { No } \\
\text { catalyst }\end{array}$ & EtOH & Reflux & 10 & - \\
& 5 & EtOH & Reflux & 12 & 40 \\
3 & 10 & $\mathrm{MeOH}$ & Reflux & 10 & 30 \\
4 & 10 & $\mathrm{CH}_{3} \mathrm{CN}$ & Reflux & 10 & 40 \\
5 & 10 & $\mathrm{DCM}^{2}$ & Reflux & 10 & 40 \\
6 & 10 & $\mathrm{CHCl}_{3}$ & Reflux & 10 & 35 \\
7 & 10 & $\mathrm{DMF}$ & Reflux & 10 & 40 \\
8 & 10 & $\mathrm{H}_{2} \mathrm{O}$ & Reflux & 10 & 50 \\
9 & 10 & EtOH $: \mathrm{H}_{2} \mathrm{O}$ & Reflux & 10 & 55 \\
10 & 20 & EtOH & $50{ }^{\circ} \mathrm{C}$ & 7 & 93
\end{tabular}

For nanostructured carbon materials like GONPs, the weak adsorption sites are characteristically placed with their basal planes consisting of the hexagonal arrangement of carbon atoms, while the high energy sites are typically associated at the edges, which are often saturated with hydrogen atoms attached to heteroatoms. The edge hydrogen atoms can be replaced by other heteroatoms, e.g. oxygen and/or nitrogen, to provide strong chemical relativities for redox or acid-base reactions. ${ }^{37}$ The GONPs catalyzed Knoevenagel condensation and Michael addition reaction supports the present mechanistic pathway in the synthesis of BCDs. ${ }^{38,39}$ As we mentioned before GONPs which has specific area $\sim 5.15 \mathrm{~m}^{2} \mathrm{~g}^{-1}$, mean pore diameter $\sim 4.6 \mathrm{~nm}$ with pore volume of $\sim 0.0876 \mathrm{~cm}^{3} \mathrm{~g}^{-1}$ (ref. 31 ) and this surfaces of the GONPs play an important role in these MCRs. The active sites i.e. hydroxy, carboxyl, epoxide etc. ${ }^{33,34}$ fulfill the requirement of acidic sites for the reaction to occur in GONPs. A plausible reaction mechanism for the GONPs catalyzed synthesis of BCDs is given in Scheme 3. The carbonyl groups in the ethyl acetoacetate are initially activated by acidic functional groups of the catalyst to react with phenylhydrazine to form pyrazolone (I). Then, the activated aldehydes by acidic functional groups of the catalyst react to form the Knoevenagel product. Subsequently, during the Michael addition step, nucleophilic attack on (II) by tautomeric form of pyrazolone (I) affords the desired product (5) via intermediate (III). Alternatively, there is another possible reaction pathway for the reaction, via the formation of intermediate (IV) followed by reaction

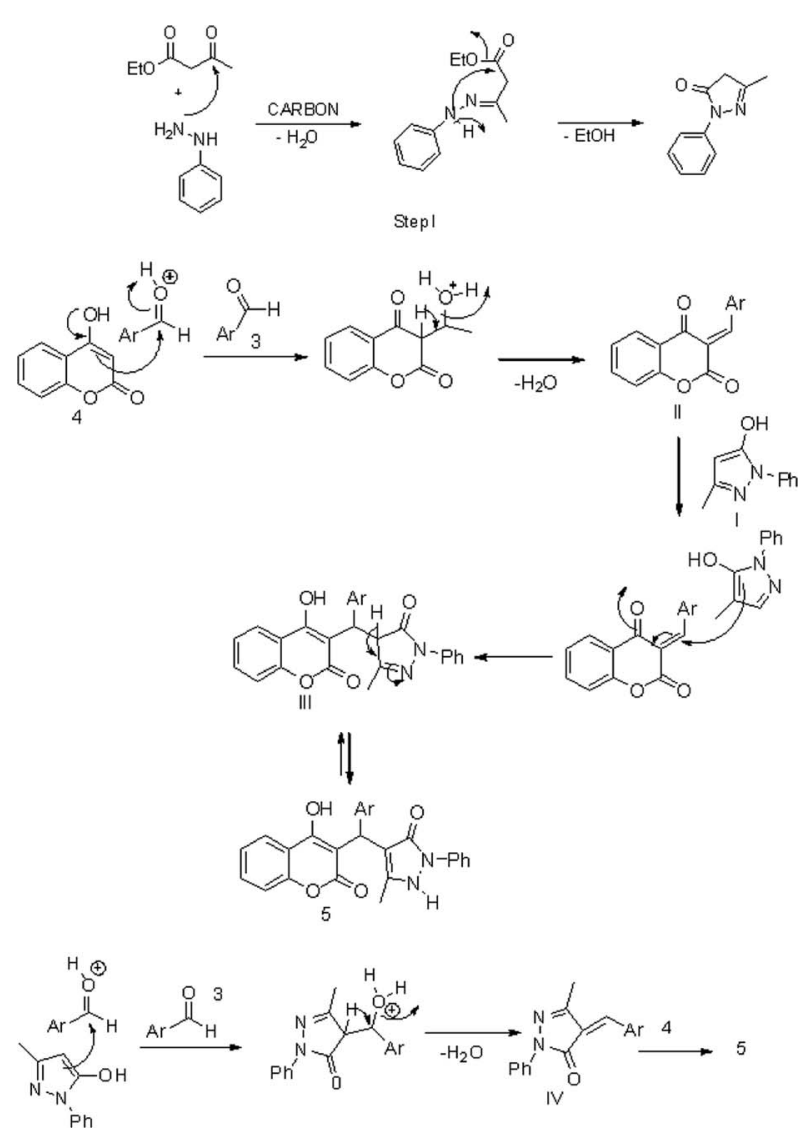

Scheme 3 Plausible mechanism for the formation of BCDs. 


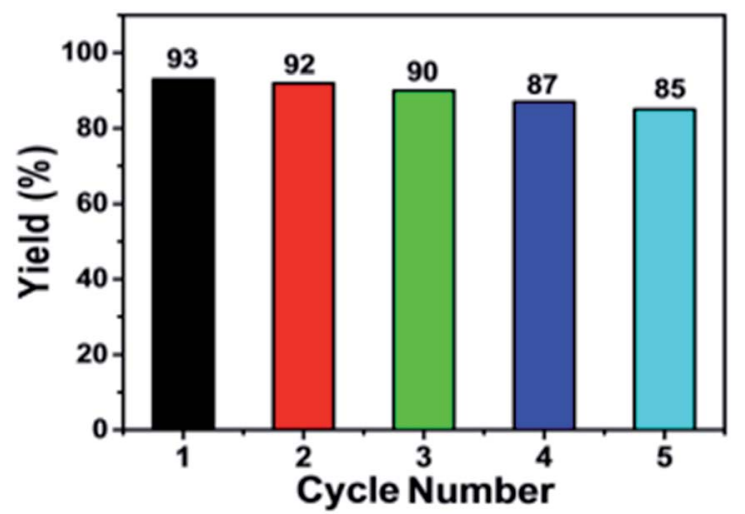

Fig. 2 Reproducibility test of GONPs for BCDs synthesis.

with 4-hydroxycoumarin to afford product (5) (see Scheme 2). It was revealed that intermediates (II) and (IV) were very reactive towards the subsequent reaction with pyrazolone and 4hydroxycoumarin respectively. ${ }^{27}$

In the multicomponent reactions proceeded through intermediate (II) or (IV) could not be measurably detected in current study. After completion of the reaction, tautomerisation occurred in the pyrazolone ring of product (5). This may exist in three tautomeric forms viz. the $\mathrm{CH}, \mathrm{OH}$ and $\mathrm{NH}^{27-30,40,41}$ The GONPs catalyst has both non-toxic and reusable features. Thus, the present protocol fulfills the criteria for green-synthesis and reusability. The robustness of GONPs for the synthesis of BCDs was tested by scrutinizing the effect of the phase and its reusability. ${ }^{17-19}$ When the synthesis of BCDs was carried out using the phases and active sites, the GONPs play significant role in catalytic activity. Finally, the GONPs, being a heterogeneous catalyst, can be easily separated from the reaction mixture and reused five times successfully (Fig. 2), by reuse of GONPs for the model reaction.

\section{Conclusion}

In conclusion, the present protocol has several features such as; (i) the prepared GONPs are nanocrystalline and mesoporous with acidic functional groups, (ii) the GONPs demonstrate a superior catalytic performance with as high as $93-82 \%$ isolated product yields, (iii) unveiling report of the biologically important highly functionalized BCDs using a solid, metal-free, nontoxic and reusable heterogeneous green/eco-friendly acid catalyst. A facile one-pot multi-component protocol has been demonstrated for the synthesis of biologically important, highly functionalized BCDs. The reaction was performed in ethanol solvent at $50{ }^{\circ} \mathrm{C}$ using GONPs as a heterogeneous acid catalyst. The products were purified by recrystallization from ethanol and chromatography-free separation methods, where the use of volatile and hazardous solvents, generally preferred, is avoided. These GONPs should find several applications in MCRs leading to biologically potent molecules in the near future, which is a topic of ongoing work.

\section{Conflicts of interest}

There are no conflicts to declare.

\section{Acknowledgements}

This work was supported by; (a) the Global Frontier Program through the Global Frontier Hybrid Interface Materials (GFHIM) of the National Research Foundation of Korea (NRF) funded by the Ministry of Science, ICT \& Future Planning 10 (2013M3A6B1078874), (b) Science Foundation Ireland under grant no. 14/IA/2581 and by the Irish Research Council under award GOIPD/2016/575, and (c) SFS would like to thank University Grants Commission, New Delhi for awarding D. S. Kothari Post-Doctoral Fellowship scheme (F.4-2/2006(BSR)/CH/ 16-17/0015).

\section{Notes and references}

1 A. T. Bell, Science, 2003, 299, 1688-1691.

2 D. Pla and M. Gómez, ACS Catal., 2016, 6, 3537-3552.

3 L. Chng, N. Erathodiyil and J. Y. Ying, Acc. Chem. Res., 2013, 20, 1825-1837.

4 H. Song, Acc. Chem. Res., 2015, 48, 491-499.

5 J. A. Torres, F. G. E. Nogueira, M. C. Silva, J. H. Lopes, T. S. Tavares, T. C. Ramalho and A. D. Corrêa, $R S C A d v$, 2017, 7, 16460-16466.

6 Y. Yong, R. Su, X. Liu, W. Xu, Y. Zhang, R. Wang, P. Ouyang, J. Wu, J. Ge and Z. Liu, Biochem. Eng. J., 2018, 129, 26-32.

7 Q. Husain, J. Nanosci. Nanotechnol., 2018, 18, 486-499.

$8 \mathrm{~J}$. B. Bharate, S. B. Bharate and R. A. Vishwakarma, ACS Comb. Sci., 2014, 16, 624-630.

9 D. R. Dreyer, H. P. Jia and C. W. Bielawski, Angew. Chem., Int. Ed., 2010, 49, 6813-6816.

10 C. Su, M. Acik, K. Takai, J. Lu, S. Hao, Y. Zheng, P. Wu, Q. Bao, T. Enoki, Y. J. Chabal and K. P. Loh, Nat. Commun., 2012, 3, 1298-1307.

11 P. T. Anastas and M. M. Kirchhoff, Acc. Chem. Res., 2002, 35, 686-694.

12 D. L. Trimm, Appl. Catal., A, 2001, 212, 153-160.

13 Y. Li and W. Shen, Chem. Soc. Rev., 2014, 43, 1543-1574.

14 I. Fechete, Y. Wang and J. C. Vedrine, Catal. Today, 2012, 189, 2-27.

15 G. V. Smith and F. Notheisz, Heterogenous Catalysis in Organic Chemistry, Academic Press, New York, 1999.

16 F. Hu, M. Patel, F. Luo, C. Flach, R. Mendelsohn, E. Garfunkel, H. He and M. Szostak, J. Am. Chem. Soc., 2015, 137, 14473-14480.

17 S. Navalon, A. Dhakshinamoorthy, M. Alvaro and H. Garcia, Chem. Rev., 2014, 114, 6179-6212.

18 D. Deng, L. Xiao, I. Chung, I. S. Kim and M. Gopiraman, ACS Sustainable Chem. Eng., 2017, 5, 1253-1259.

19 C. L. Su and K. P. Loh, Acc. Chem. Res., 2013, 46, 2275-2285. 20 D. S. Su, J. Zhang, B. Frank, A. Thomas, X. Wang, J. Paraknowitsch and R. Schlogl, ChemSusChem, 2010, 3, 169-180.

21 M. J. Allen, V. C. Tung and R. B. Kaner, Chem. Rev., 2010, 110, 132-145.

22 W. S. Hummers and R. E. Offeman, J. Am. Chem. Soc., 1958, 80, 1339. 
23 A. Lerf, H. Y. He, M. Forster and J. Klinowski, J. Phys. Chem. $B, 1998,102,4477-4482$.

24 T. Nakajima and Y. Matsuo, Carbon, 1994, 32, 469-475.

25 J. H. Clark, Acc. Chem. Res., 2002, 35, 791-797.

26 R. A. Sheldon, Green Chem., 2005, 7, 267-278.

27 P. P. Ghosh, G. Pal, S. Pal and A. R. Das, Green Chem., 2012, 14, 2691-2698.

28 A. Saha, S. Payra and S. Banerjee, Green Chem., 2015, 17, 2859-2866.

29 B. Karami, K. Eskandari, S. Khodabakhshi, S. S. Hoseini and F. Hashemian, RSC Adv., 2013, 3, 23335-23342.

30 S. Yaragorla, A. Pareek and R. Dada, Tetrahedron Lett., 2015, 56, 4770-4774.

31 B. G. Ghule, S. Shaikh, S. U. Ekar, U. T. Nakate, K. C. Gunturu, N. M. Shinde, M. Naushad, K. H. Kim, C. O'Dwyer and R. S. Mane, ACS Appl. Mater. Interfaces, 2017, 9, 43051-43060.

32 Y. Li, Y. Hu, H. Li, L. Chen and X. J. Huang, J. Mater. Chem. A, 2016, 4, 96-104.

33 J. Paredes, S. Villar-Rodil, A. Martinez-Alonso and J. Tascon, Langmuir, 2008, 24, 10560-10564.

34 B. Garg, T. Bisht and Y. C. Ling, Molecules, 2014, 19, 1458214614.
35 M. Patel, W. Feng, K. Savaram, M. R. Khoshi, R. Huang, J. Sun, E. Rabie, C. Flach, R. Mendelsohn, E. Garfunkel and H. He, Small, 2015, 11, 3358-3368.

36 P. L. Chiu, D. D. T. Mastrogiovanni, D. Wei, C. Louis, M. Jeong, G. Yu, P. Saad, C. R. Flach, R. Mendelsohn, E. Garfunkel and H. He, J. Am. Chem. Soc., 2012, 134(13), 5850-5856.

37 L. R. Radovic and F. Rodriguez-Reinso, Carbon Materials in Catalysis. Chemistry and Physics of Carbon: A Series of Advances, Marcel Dekker, Inc., New York, 1997.

38 S. Verma, H. P. Mungse, N. Kumar, S. Choudhary, S. L. Jain, B. Sain and O. P. Khatri, Chem. Commun., 2011, 47, 1267312675.

39 S. M. Islam, A. S. Roy, R. C. Dey and S. Paul, J. Mol. Catal. A: Chem., 2014, 394, 66-73.

40 V. I. Minkin, L. P. Olekhnovic and Y. A. Zhdanov, Molecular Design of Tautomeric Compounds, Reidel, Dordrecht, The Netherlands, 1988.

41 D. H. Barton, Comprehensive Organic Chemistry: The Synthesis and Reactions of Organic Compounds, Pergamon Press, Oxford, U.K., 1979, vol. 4 Heterocyclic Compounds, 2698. 